nen Angaben bereits seit dem "Symposium des Arbeitskreises Junger Kriminologen« I97I, auf dem er seinen »marxistisch-interaktionistischen Ansatz " vortrug, kaum noch wissenschaftlicher sondern vorrang politischer Kritik (Vorwurf des Extremismus, wobei unklar ist, ob Links- oder Rechtsextremismus gemeint ist) seitens der Fachkollegen ausgesetzt. ${ }^{9}$ Dabei ist Sack gewiß kein Marx-Epigone, sondern er entwickelt die marx'sche Position - schöpferisch? - weiter. Wie weiland Marx den Hegel, so hebt auch Sack die marx'sche Position dialektisch auf, stellt sie wieder auf den Kopf. Nicht der Verbrecher produziert den Richter, den Schergen, sondern nach Sack produziert der Scherge, der Richter, den Verbrecher: "Was sich in den Kriminalstatistiken ... niederschlägt, das sind ja die Ergebnisse von Verfahren, ... die die Eigenschaft (Kriminalität, Delinquenz), über die das spätere Urteil eine deskriptive Aussage macht, tatsächlich erst begründen und ins Leben rufen ". ${ }^{10}$

Trotz aller Anfeindungen halten junge Kriminologen weiterhin an Marx fest. In seiner Arbeit »Kriminalität ist normal« findet Haferkamp 1972 den Mut, die "Entökonomisierung der Soziologie und speziell der Soziologie des abweichenden Verhaltens «11 zu bedauern und betont, daß die Situationsdefinitionen der Kontrollinstanzen unschwer »mit den Ansprüchen des Wirtschaftssystems moderner Gesellschaften ${ }^{12}$ zu vermitteln seien. Offensichtlich eingeschüchtert, leistet er diese Vermittlung jedoch nicht mehr.

Dennoch: Im »Kriminologischen Journal« Heft $3 / 72$ geht man sogar soweit, einen Aufsatz von Hess "Die Lazarusschicht - Vorbemerkungen zum Problem der Armut « ausdrücklich mit der Begründung abzudrucken: »..., weil er das Problem der ,Armut unter Bedingungen kapitalistischer Arbeits- und Verwertungsprozesse stellt $\ll .{ }^{13}$

Sicher, diese Marx-Rezeption ist nicht aufzuhalten. Nur ist es der Satiriker Marx, der hier rezipiert wurde, nicht der Wissenschaftler. Daß die bürgerliche Wissenschaft sich in einer glossierenden Abschweifung wiedererkennt und damit ein Kapitel schwarzen Humors in den wissenschaftlichen Himmel hebt, ist nun selbst ein hübsches Beispiel unfreiwilliger Ironie.

Falco Werkentin

\title{
Spendenaufruf des Initiativkomitees "Solidarität für Günter Amendt«
}

Die Auftragsdruckerei des Springerkonzerns will später Rache nehmen. Sie hat ein rechtskräftiges BGH-Urteil gegen Günter Amendt erwirkt, das ihn als einzelnen aus der Masse einer Demonstration herausgreift und für ihn zur Folge hat, daß er DM 72000 zahlen soll. Das soll ihn in seiner bürgerlichen Existenz vernichten (indirektes Berufsverbot). In Wirklichkeit waren die Aktionen Ostern r 968 gegen die Auslieferung der Springerzeitungen eine kollektive und spontane Antwort auf die monatelange Hetze in den Springerblättern gegen die außer-

\footnotetext{
9 vgl. F. Sads $\times$ Definition von Kriminalität als politisches Handeln: der labeling approache, in: Kriminologisches Journal, Heft $1 / 1972$ bes. S. 4.

10 cbenda, S. 2 I.

11 Stuttgart 1972, S. 136.

12 ebenda, S. 107.

13 S. I6I.
} 
parlamentarische Opposition, der das Attentat auf Rudi Dutschle folgte. Die Absicht der Druckerei, ihr Vergeltungsbedürfnis an einem einzelnen zu befriedigen, ist bezeichnend. Wir vereiteln die Verwirklichung dieser Absicht, indem wir die eingeklagten $D M 72000$ durch eine Sammelaktion aufbringen.

Auf Aufforderung des Initiativkomitees begründet Günter Amendt, warum er die Solidaritätsaktion für politisch wichtig hält.

„Die Argumente der Genossen haben mich von der politischen Notwendigkeit überzeugt, eine breite Solidarisierung zu organisieren. Aus diesem Grunde bin ich bereit, aktiv alle Initiativen zu unterstützen und politisch zu vertreten, die versuchen, die Konsequenzen dieses Urteils zu tilgen und so uns allen den Beweis liefern, daßß die Strategie der Klassenjustiz an der massenhaften Solidarität der Genossen scheitert. Mit dieser politischen Reaktion ist mir aus der persönlichen Misere geholfen. Denn als einzelner bin ich nicht bereit, die Summe zu zahlen, zu der ich verurteilt bin. Ich kann es nicht, und ich will es nicht. Die verschiedenen Hilfsangebote haben mich in diesem Entschluß bestätigt. Ich mache mir keine Illusionen über die Konsequenzen einer solchen Weigerung. Die ständige Verfolgung durch Vollstreckungsbeamte der Justiz stellt für einen einzelnen eine katastrophale Bedrohung der persönlichen - und damit verbunden - auch jeder politischen Perspektive dar. Praktisch liefe die Vollstredkungsdrohung auf ein $>$ kaltes Berufsverbot $<$ hinaus.

Aber selbst das Angebot noch so günstiger Abzahlungsbedingungen wäre, akzeptiert man es, eine unzulässige Kumpanei mit der Klassenjustiz und der Springerbande, deren Handlanger sie ist. Als Verurteilter die Rechnung zu begleichen, käme, um es drastisch zu sagen, einem Verrat an einer Phase des politischen Kampfes gleich, von deren historischer Notwendigkeit ich damals wie heute überzeugt bin. Ich bin überhaupt nicht berechtigt, durch eine Abzahlung die Bedeutung der Springer-Kampagne zu liquidieren, nur weil ein Gerichtsurteil mich dazu zwingen will. Genau aus diesem Grund haben der Genosse Krahl und ich in einer früheren Phase des Prozesses einen Vergleich mit Springers Auftragsdruckerei abgelehnt. Krahl und ich sind - obwohl von Anfang an von der Erfolglosigkeit des juristischen Weges überzeugt - sehr weit gegangen, als wir uns trotzdem darauf einließen, alle Wege der bürgerlichen Justiz zu gehen und damit die Mittel des bürgerlichen >Rechtsstaates bis zur letzten Instanz auszuloten. Das Ergebnis ist bekannt und bestätigt unsere Erwartungen an das Funktionieren der Justiz, wenn es darum geht, die Interessen der Herrschenden wahrzunehmen. Wer jetzt noch immer die Auffassung vertritt, noch seien nicht alle Mittel erschöpft, noch stünde der Weg einer Verfassungsklage offen, der überstrapaziert den Glauben an die Möglichkeiten, die Kommunisten und Sozialisten unter den Bedingungen dieser Gesellschaft gegeben sind. Denn schließlich ist das Urteil eingebettet in den forcierten Abbau demokratischer Rechte in allen Bereichen. Weil der Kampf gegen eine verschärfte Entdemokratisierung eine wesentliche Aufgabe aller sozialistischen und demokratischen Kräfte ist, wird die solidarische Liquidierung dieses Urteils politisch notwendig.

Die Bereitschaft der Genossen, sich an einer solchen Aktion zu beteiligen, resultiert fast übereinstimmend aus der Bedeutung, die sie den Aktionen gegen Springer beimessen, und die viele als eine wesentliche Voraussetzung ihrer heutigen politischen Arbeit betrachten. Wenn auch die politisch organisatorischen Konsequenzen, die die einzelnen Gruppen aus ihrer ,Frühzeit< der Studentenbewegung bezogen haben, sehr unterschiedlich sind, so war in allen Diskussionen die ehemalige Aktionseinheit bestimmendes Moment, sich jetzt an einer solchen Aktionseinheit zu beteiligen. 
Einen in diesen Diskussionen auftauchenden Einwand und die Antwort auf ihn, will ich hier nicht auslassen. Einige Genossen haben abgewinkt, als der Plan diskutiert wurde, durch eine Sammelaktion das Urteil zu beantworten. Man kann doch der Druckerei nicht 72000 DM in den Rachen werfen, so lautete das Argument. Wenn man so argumentiert, dann unterschätzt man die Kapitalkraft der Auftragsdruckerei des Springer-Konzerns. Selbstverständlich ist es für die Drukkerei egal, ob sie das Geld bekommt oder nicht. Ihr geht es um ein Exempel, das abschreckende Wirkung haben soll. Für uns kann es einzig darauf ankommen, will man die 72000 DM bewerten, die in dieser Summe sreckende Arbeitskraft $\mathrm{zu}$ sehen, die der Verurteilte als Lohnsklave für die Druckerei aufbringen soll bis die Schulden getilgt sind. Genau darin steckt die Zumutung und genau hierin begründet sich die Weigerung, als Einzelperson zu zahlen. Eine Sammlung ist auch nur deshalb gerechtfertigt, weil sich die Gesamtsumme aus vielen Einzelleistungen zusammensetzt, die, gemessen an der politischen Bedeutung des Urteils, politisch vertretbar sind.

Einige Genossen haben erklärt, daß sie zwar prinzipiell bereit seien, eine Sammlung aktiv zu unterstützen, sie schlagen aber vor, so lange zu warten, bis man mir - als dem Verurteilten - die Zahlungsaufforderung präsentiert. Hinter diesem Vorschlag steht die Hoffnung, daß die Drudkerei es gar nicht wage, den Verurteilten zur Zahlung zu zwingen. Leider verkennt ein solcher Vorschlag die Tragweite eines Zivilrechtsurteils. Es ist durchaus möglich, daß sich die Druckerei aus politischen Gründen mit der Eintreibung Zeit läßt. Und sie kann sich das leisten. Sie kann es sich, genau gesagt, 30 Jahre lang leisten, denn so lange verfügt sie über diesen Rechtstitel, und es ist naheliegend, daß sich die Druckerei einen Zeitpunkt aussucht, an dem sie annehmen kann, daß - im zeitlichen $\mathrm{Ab}$ stand - die massenhafte Solidarisierung, die heute möglich ist, dann nicht mehr zustandekommt."

Spenden sind zu überweisen an Solidaritäts-Sonderkonto Günter Amendt, Girokonto 1743996I-I I bei Bank für Gemeinwirtschaft Niederlassung Offenbach; das Postscheckkonto der Bank: Postscheckamt Frankfurt 172480. Kontostand Mitte November 1972: 24000 DM. 